\title{
miR-212 and miR-132 are required for epithelial stromal interactions necessary for mouse mammary gland development
}

\author{
Ahmet Ucar ${ }^{1,5}$, Vida Vafaizadeh ${ }^{2}$, Hubertus Jarry ${ }^{3}$, Jan Fiedler ${ }^{4}$, Petra A B Klemmt ${ }^{2}$, Thomas Thum ${ }^{4}$, \\ Bernd Groner ${ }^{2} \&$ Kamal Chowdhury ${ }^{1}$
}

\begin{abstract}
MicroRNAs are small noncoding RNAs that carry out post-transcriptional regulation of the expression of their target genes. However, their roles in mammalian organogenesis are only beginning to be understood. Here we show that the microRNA212/132 family (which comprises miR-212 and miR-132) is indispensable during the development of the mammary glands in mice, particulary for the regulation of the outgrowth of the epithelial ducts. Mammary transplantation experiments revealed that the function of the miR-212/132 family is required in the stroma but not in the epithelia. Both miR-212 and miR-132 are expressed exclusively in mammary stroma and directly target the matrix metalloproteinase MMP-9. In glands that lack miR-212 and miR-132, MMP-9 expression increases and accumulates around the ducts. This may interfere with collagen deposition and lead to hyperactivation of the tumor growth factor- $\beta$ signaling pathway, thereby impairing ductal outgrowth. Our results identify the miR-212/132 family as one of the main regulators of the epithelial-stromal interactions that are required for proper pubertal development of the mammary gland.
\end{abstract}

MicroRNAs are endogenous regulatory small RNAs that associate with the $3^{\prime}$ untranslated regions (UTRs) of their target mRNAs and cause their degradation or translational inhibition ${ }^{1,2}$. MicroRNA-mediated gene regulation is crucial for many biological processes such as cellular growth, differentiation, mobility and death ${ }^{3}$. However, in contrast to fruit flies and nematodes, the functional importance of microRNAs during vertebrate development is only beginning to be analyzed ${ }^{4}$.

The miR-212/132 family is highly conserved in vertebrates. MiR132 regulates neuronal morphogenesis and the dendritic plasticity of cultured neurons ${ }^{5-8}$. Moreover, antagomir-mediated knockdown studies in adult mice showed that miR-132 regulates some aspects of the circadian clock ${ }^{9}$ and inflammatory pathways ${ }^{10}$. We used deletion mutagenesis to investigate the developmental role(s) of miR-212 and miR-132 in the mouse and found that their absence leads to a severe impairment of mammary gland development.

The mammary gland has unique features, as most of its development takes place during postnatal life ${ }^{11-14}$. In mice, an epithelial placode is formed during embryogenesis. Upon birth, a rudimentary ductal structure is present in the nipple area. During pubertal development, these ducts extend throughout the fat pad until they reach the peripheries of the gland. This invasive growth of the ducts occurs at their distal tips, called terminal end buds (TEBs). Additional ductal side branches are also formed during repeated estrus cycles. During pregnancy, these ductal structures differentiate into lobuloalveolar structures, which produce and secrete milk during the lactation period. After weaning, the mammary gland involutes and looks similar to an adult virgin mammary gland.

The mammary gland of a virgin mouse consists of two separate compartments: the epithelial ducts and the surrounding stroma ${ }^{15,16}$. Although epithelial ductal outgrowth is the most obvious macroscopically visible structural change during pubertal mammary gland development, the stroma has crucial functions in the induction and regulation of this ductal outgrowth by providing essential growth factors and extracellular matrix (ECM) proteins ${ }^{16-18}$. Here we show that the function of miR-212 and miR-132 in the mammary stroma is indispensable for the regulation of epithelial-stromal interactions that are required for ductal outgrowth during pubertal development of the mammary glands.

\section{RESULTS}

Generation of mice lacking miR-212 and miR-132

miR-212 and miR-132 have been reported to be generated from a stable intron of a non-protein coding gene that is expressed in primary neuronal cultures ${ }^{5,19}$. However, we identified a new transcript variant with different exon-intron structures that encoded both microRNAs in its second exon (Supplementary Fig. 1a). These two transcript variants obviously represent different alternatively spliced forms of the gene ( $m i R-212 / 132)$ that encodes miR-212 and miR-132.

\footnotetext{
${ }^{1}$ Department of Molecular Cell Biology, Max Planck Institute of Biophysical Chemistry, Goettingen, Germany. ${ }^{2}$ Georg-Speyer-Haus, Institute for Biomedical Research, Frankfurt am Main, Germany. ${ }^{3}$ Department of Endocrinology, University of Goettingen, Goettingen, Germany. ${ }^{4}$ Institute for Molecular and Translational Therapeutic Strategies, Hannover Medical School, Germany. ${ }^{5}$ Present address: Cellular Senescence Group, German Cancer Research Center (DKFZ), Heidelberg, Germany. Correspondence should be addressed to K.C. (kchowdh@gwdg.de).
} 
Figure 1 Genetic deletion of miR-212/132 in mice results in mammary gland defects associated with impaired nourishment of pups. (a) Pup growth curve analysis. Pups of homozygous mutant females had retarded growth. The differences in body weights of miR-212/132 $2^{-1-}$ mothers' pups were significant at postnatal day $2(P<0.01)$ and at all subsequent days $(P<0.001)$ compared with pups of wild-type mothers. No significant difference was observed between the growth curves for pups of wild-type or heterozygous mothers. Three litters per genotype were used for this analysis. Data are expressed as mean \pm s.d.; $n=15$ for each group. (b) Neutral-red staining of sections from lactating mammary gland samples. The dense epithelial staining (red) was seen throughout the whole section of the glands from wild-type and heterozygous mice. Epithelia within homozygous mutant glands were observed only in the proximal region close to the nipple. The boundaries of the section for the homozygous mutant sample are shown with dashed lines, as the non-epithelial fat pad does not show any staining.

Isoform 1 is expressed only in the testes and brain, whereas isoform 2 is expressed in the brain, testes, heart and mammary glands (Supplementary Fig. 1b).

To investigate the physiological and developmental roles of miR-212 and miR-132 in mice, we used homologous recombination to delete the genomic region that encodes pre-miR-212 and pre-miR-132 (Supplementary Fig. 2a). DNA blotting (Supplementary Fig. 2b) and quantitative RT-PCR analyses (data not shown) confirmed successful genomic deletion and the complete loss of both microRNAs in homozygous mutants.

miR-212/132 $2^{-/-}$mice were born with the expected Mendelian ratio and had a normal life span. However, although the female miR-212/132-/mice showed normal nursing behaviors, their pups had severe growth problems (Fig. 1a) and usually died within 5 days of birth. The survival rate of these pups was inversely proportional to the litter size (data not shown). Cross-fostering experiments showed that wild-type females' pups faced the same problems under the care of $m i R-212 / 132^{-/-}$mothers, whereas the pups of $m i R-212 / 132^{-/-}$females grew and survived normally if they were fostered by wild-type mothers (data not shown). Thus, miR-212/132-I- female mice had difficulty in feeding their pups.

To identify the reason for this difficulty, we examined lactating mammary glands histologically (Fig. 1b). In contrast to wild-type or heterozygous glands, $m i R-212 / 132^{-l-}$ glands had an abnormal appearance. Dense lobuloalveolar structures were restricted to the regions close to the nipple area, and the rest of the fat pads had no epithelial structures.

\section{Impaired ductal outgrowth in $\mathbf{m i R}-212 / 132^{-/-}$glands}

We characterized the observed phenotype further by whole-mount staining of the mammary glands of pubertal, pregnant and lactating mice (Fig. 2 and Supplementary Fig. 3). The rudimental ductal trees appeared normal in both wild-type and $m i R-212 / 132^{-/-}$pre-pubertal mice (Fig. 2a,b). At 5 weeks of age, the ductal structures reached the region of the lymph nodes in wild type glands and TEBs were visible at their distal ends (Fig. 2c). However, the rudimental ductal trees in
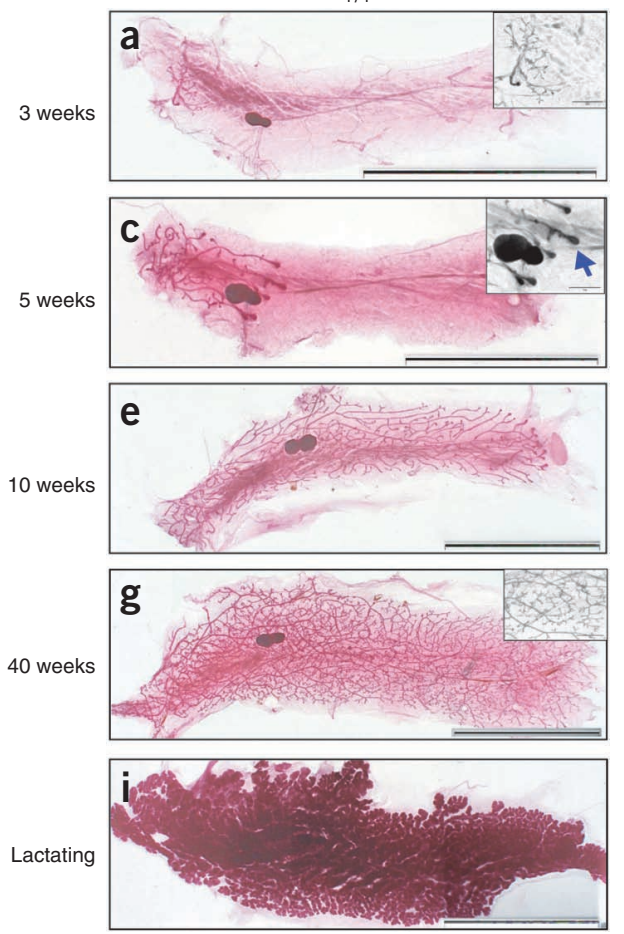
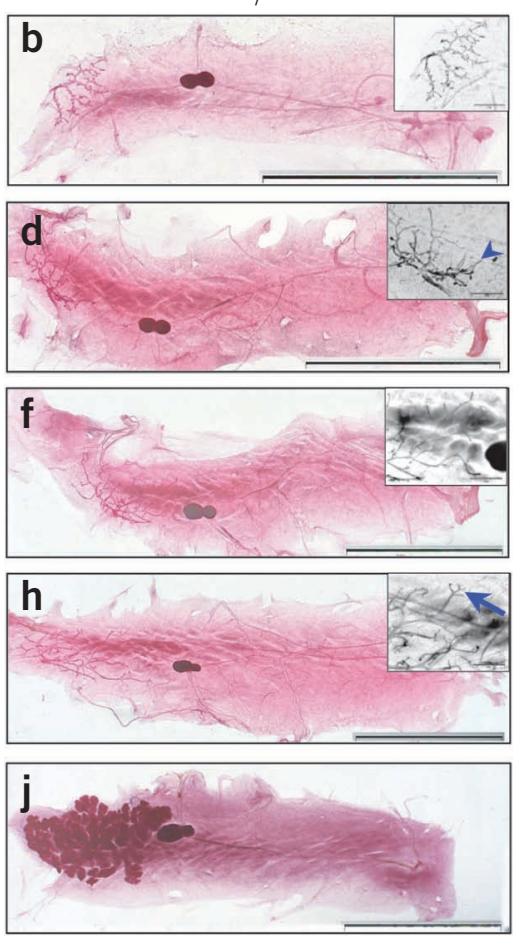

Figure 2 miR-212/132-/- mammary glands have a defect in pubertal ductal outgrowth, but not in ductal side branching and lobuloalveolar differentiation. (a-j) Whole-mount analyses of inguinal (no. 4) mammary glands from wild-type and homozygous mutant littermates at different stages of mammary gland development. Virgin stages are pre-pubertal (3 weeks; a,b), early pubertal (5 weeks; c,d), late pubertal (10 weeks; e,f), and 40-week-old adult (g,h). Lactating samples (i,j) were obtained from mice killed 1 day after giving birth. Proximal-to-distal orientations of all samples are shown as left-to-right. The grayscale insets are highmagnification pictures of corresponding samples for detailed visualization. In wild-type glands, TEBs were formed (arrow in c) and ductal outgrowth took place during puberty $(\mathbf{c}, \mathbf{e})$. No TEBs were observed at the distal ends of the ducts in mutant glands (arrowhead in d) and ductal outgrowth did not take place $(\mathbf{d}, \mathbf{f})$. Ductal side branching was observed in mutant glands (arrows in $\mathbf{h}$ ) similar to wild-type glands (g). Lobuloalveolar structures were formed in mutant glands during pregnancy and milk-producing alveoli were seen during lactation (j) similar to wildtype glands (i), but restricted to the proximal region where the rudimentary ductal tree was located. At least five mice were analyzed for each genotype at each stage. Scale bars represent $1 \mathrm{~cm}$; for grayscale insets, scale bars represent $1 \mathrm{~mm}$. 
Figure 3 miR-212/132-/- mammary epithelia have normal ductal architecture. (a-f) Mammary gland sections from 6-week-old wild-type $(\mathbf{a}, \mathbf{c}, \mathbf{e})$ and homozygous mutant $(\mathbf{b}, \mathbf{d}, \mathbf{f})$ littermates were analyzed by hematoxylin-eosin (H\&E) staining $(\mathbf{a}, \mathbf{b})$, and immunohistochemistry for E-cadherin (E-cadh; c,d) and cytokeratin 14 (CK-14; e,f). E-cadherin is a marker for luminal epithelial cells, whereas cytokeratin-14 is expressed only by myoepithelial cells within the ductal structures. Both wild-type and homozygous mutant mammary ducts have an inner single layer of luminal epithelial cells and outer layer of myoepithelial cells, as shown by E-cadherin and cytokeratin-14 staining, respectively. Fibroblasts are distinguishable within the periductal stroma for both genotypes. Scale bars represent $100 \mu \mathrm{m}$.

miR-212/132-/- glands failed to initiate ductal outgrowth and produced no TEBs (Fig. 2d). At 10 weeks of age, the ductal tree in wild type littermates almost reached the end of the fat pad (Fig. 2e), but the ductal structures in mutant glands retained their pre-pubertal appearance (Fig. 2f). Wild-type glands contained elaborate epithelial structures at 40 weeks of age owing to extensive ductal side-branching that had occurred during repeated estrus cycles (Fig. 2g). miR-212/132-/glands contained similar structures, although the ductal tree did not extend much further than in the pre-pubertal stage (Fig. 2h).

The lobuloalveolar structures of mammary glands develop gradually during pregnancy and reach their mature forms by parturition (Fig. 2i). Similar lobuloalveolar structures also develop in $m i R-212 / 132^{-/-}$ glands, but they extend through a much smaller portion (only up to $20 \%$ ) of the fat pad (Fig. 2 j and Supplementary Fig. 3). Thoracic mammary glands showed similar phenotypes (data not shown). It seems reasonable that this difference accounts for the decrease in milk production and could explain the growth and survival problems of the pups of homozygous mutant females.

\section{Mutant ducts lack TEB but retain the capacity for outgrowth}

To determine whether there is a structural defect in $m i R-212 / 132^{-/-}$ epithelial ducts, we analyzed the pubertal mammary glands histologically. We found several typical TEB structures in wild-type glands but not in $m i R-212 / 132^{-1-}$ glands (data not shown). On the other hand, the ductal structures of both genotypes looked normal with an inner layer of luminal epithelium and an outer layer of myoepithelium (Fig. 3a,b). Immunostaining for E-cadherin and cytokeratin-14 confirmed the identity of luminal epithelial (Fig. 3c,d) and myoepithelial (Fig. 3e,f) cells, respectively. Both genotypes also had fibroblasts in the periductal stroma (Fig. 3a,b).

Ductal outgrowth depends on extensive cellular proliferation of the cells in TEBs ${ }^{12-14}$. In addition, the lumen of growing ducts is generated through apoptosis of inner layers of body cells as the TEBs push the ductal outgrowth further ${ }^{20}$. As $m i R-212 / 132^{-/-}$glands seem to contain no TEBs, we evaluated the numbers of apoptotic and proliferating cells only in the ductal regions of wild-type and $m i R-212 / 132^{-/-}$glands. We found no significant difference in the number of TUNEL- or bromodeoxyuridine (BrdU)-positive cells (data not shown). This suggests that the observed phenotype is not due to a defect in proliferation or apoptosis in already formed ducts.

In $m i R-212 / 132^{-/-}$mammary glands, the isometric growth of ducts continues during puberty (Supplementary Fig. 3), although the allometric growth driven by TEBs does not take place. Therefore, the main defect in $m i R-212 / 132^{-/-}$glands should be related to the generation or stability of TEBs, or both. To find out whether the miR-212/132-/epithelia have an a priori defect in forming stable TEBs, we performed reciprocal mammary epithelial transplantation experiments.

We transplanted mammary epithelial ducts from pre-pubertal $m i R-212 / 132^{-/-}$mice into cleared fat pads of pre-pubertal wild-type

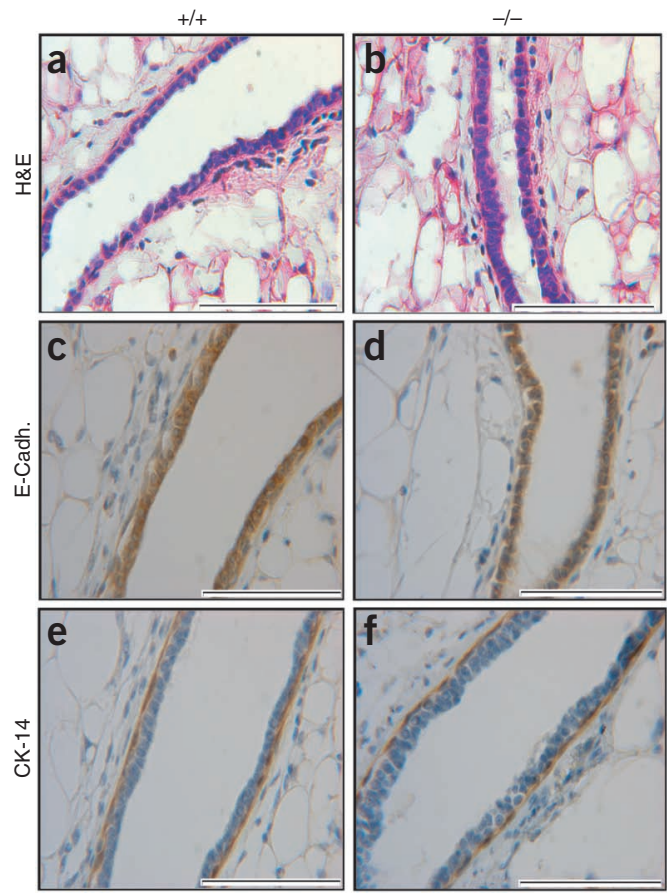

mice and vice versa (Fig. 4a). When we transplanted pre-pubertal miR-212/132-l- mammary epithelia into the cleared wild-type fat pad, they formed normal TEBs (arrows in Fig. 4b) and invaded the whole fat pad (Fig. 4c,d). Moreover, they also developed proper lobuloalveolar structures during pregnancy (Fig. 4e). By contrast, pre-pubertal wild-type mammary epithelia that were transplanted into cleared fat pads from miR-212/132-/- mice did not form TEBs (Fig. 4f) and consequently did not invade the mutant fat pads 12 weeks (Fig. 4g,h) or 25 weeks after transplantation (data not shown). However, lobuloalveolar differentiation took place during pregnancy in the limited ductal regions around the transplantation site (Fig. 4i) at a similar level as in the non-transplanted contralateral gland of miR-212/132-l- mice (data not shown).

These data suggest that the $m i R-212 / 132^{-/-}$epithelia have no apparent histological defects or inherent functional deficiencies and can form TEBs to achieve normal ductal outgrowth. Our observation that wild-type epithelia show impaired ductal outgrowth in cleared $m i R-212 / 132^{-1-}$ fat pads also indicates that either the mammary stroma or the hormonal environment of $m i R-212 / 132^{-/-}$mice is the primary cause of the impaired ductal outgrowth phenotype.

\section{Mutant stroma is non-permissive for ductal outgrowth}

From puberty, all subsequent stages of mammary gland development are under the control of endocrine systems ${ }^{11-14}$. Estrogen and growth hormones are the main endocrine regulators of the pubertal stage of mammary gland development. Therefore, we measured the serum levels of both hormones in $m i R-212 / 132^{-/-}$mice and found no significant differences when compared with female littermates of all genotypes (Supplementary Fig. 4).

As $m i R-212 / 132^{-/-}$epithelia maintain the functional capacity to undergo ductal outgrowth, and estrogen and growth hormone levels are normal in the mutant mice, the impaired ductal outgrowth in mutant mammary glands is probably due to a stromal deficiency. To verify this hypothesis, we performed whole mammary gland transplantations.

We transplanted whole mammary glands from 5-week-old donor mice into 5-week-old wild-type and $m i R-212 / 132^{-/-}$recipient mice 
a
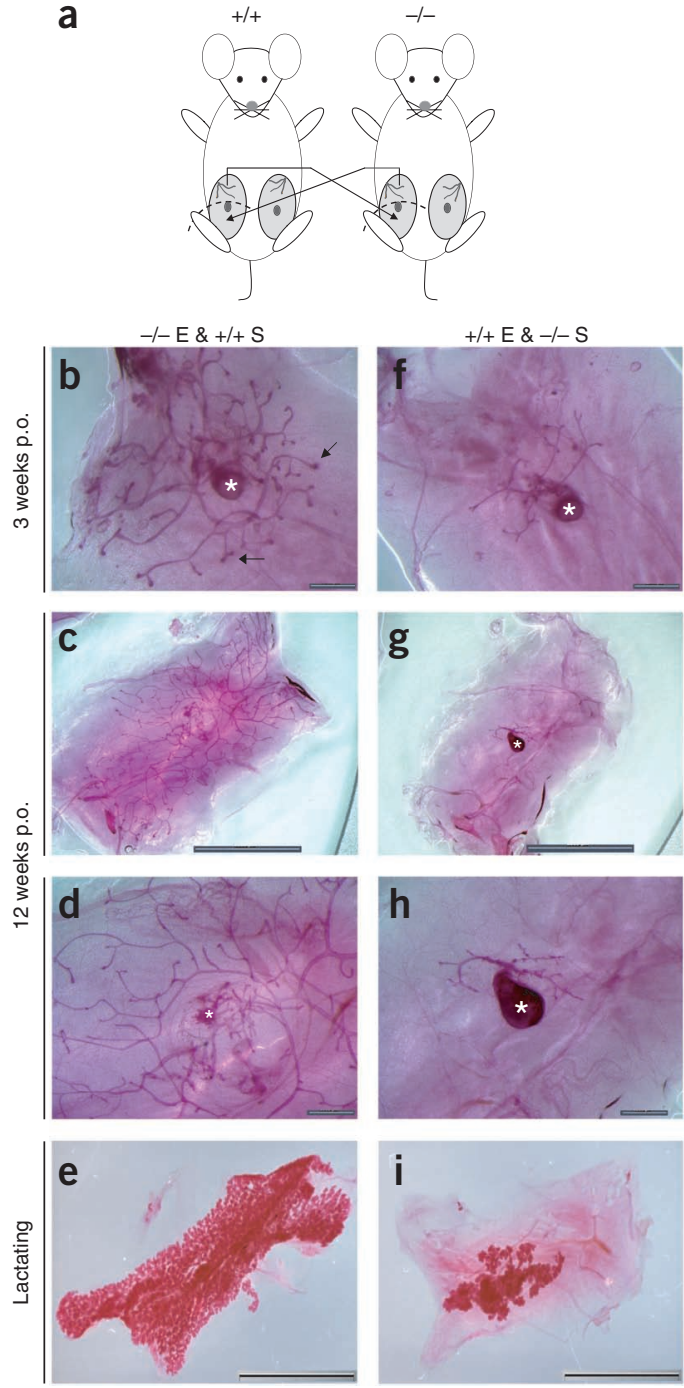

(Fig. 5) and analyzed them 5 or 10 weeks later for ductal outgrowths. Five weeks after being transplanted into either miR-212/132-/(Fig. 5a) or wild-type recipient mice (Fig. 5b), wild type or heterozygous glands contained proper TEBs and ductal outgrowth, which eventually covered the whole gland with epithelial ducts (Fig. 5c,d). By contrast, miR-212/132-- glands showed no signs of ductal outgrowth when they were transplanted into either $m i R-212 / 132^{-/-}$ (Fig. 5e) or wild-type mice (Fig. 5f).

To investigate the possibility that the mutant phenotype was caused by an earlier endocrine defect which might have an effect only during the onset of puberty, we also transplanted whole mammary glands from pre-pubertal mice into 5-week-old recipients and analyzed them 5 weeks later for the initiation of ductal outgrowth. Wild-type glands from pre-pubertal donors formed proper TEBs and showed normal ductal outgrowth in miR-212/132 ${ }^{-/-}$mice (Fig. 5g). By contrast, we found no ductal outgrowths in glands that were transplanted from pre-pubertal miR-212/132-/- donors into wild-type mice (Fig. $5 \mathbf{h}$ ). These findings clearly show that the cause of the mutant phenotype is not an endocrine defect, which could prevent the generation of TEBs during the onset of puberty.

Together, these results suggest that the cause of the impaired ductal outgrowth phenotype in miR-212/132-- mammary glands is that the mutant stroma is not permissive for pubertal outgrowth of epithelial ducts.
Figure 4 miR-212/132-/- mammary epithelia have normal capacity for ductal outgrowth. (a) The strategy of the mammary epithelial transplantation. The inguinal (no. 4) glands are shown together with rudimentary epithelial tree in the proximal region close to the nipple area. The locations of the cuts for clearing the fat pads are shown as dashed lines. The arrows show the origin of transplants and the location to which they were transplanted. (b-i) Whole-mount staining of the transplanted mammary glands analyzed 3 and 12 weeks after the operation (p.o.) and in very early lactation. Asterisks show the region where the transplants were placed during the operation. Homozygous mutant epithelia (-/-E) formed TEBs (arrow in b) and underwent proper ductal outgrowth within the wild-type fat pad stroma $(+/+S$; $\mathbf{b}-\mathbf{d})$. During pregnancy these ductal structures differentiated into lobuloalveolar structures (e). By contrast, wild-type epithelia $(+/+E)$ did not generate TEBs to invade the homozygous mutant fat pad stroma $(-/-\mathbf{S} ; \mathbf{f}-\mathbf{h})$. However, these underdeveloped ductal structures, which were restricted to the region of transplantation, underwent lobuloalveolar differentiation during pregnancy (i). $\mathbf{c}$ and $\mathbf{g}$ are low-magnification pictures of $\mathbf{d}$ and $\mathbf{h}$, respectively. Scale bars represent $1 \mathrm{~cm}(\mathbf{c}, \mathbf{e}, \mathbf{g}, \mathbf{i})$ and $2 \mathrm{~mm}(\mathbf{b}, \mathbf{d}, \mathbf{f}, \mathbf{h})$.

miR-212 and miR-132 are restricted to mammary gland stroma We used quantitative RT-PCR to determine the expression kinetics of miR-212 and miR-132 during different stages of mammary gland development (Supplementary Fig. 5). Their expression is low at the pre-pubertal stage and gradually increases throughout puberty, reaching a maximum at around 10 weeks of age, which coincides with the completion of ductal outgrowth. Although the expression of miR-212 and miR-132 substantially decreases and reaches its minimum by the end of pregnancy, it starts to increase again immediately after parturition. High variability in expression during puberty and adulthood might indicate that the expression is controlled by the estrus cycle. Indeed, this variability is much lower before puberty and during pregnancy, when the estrus cycle is not activated or has been discontinued, respectively. The expression of miR-212 and miR-132 in periovulatory granulosa cells is highly upregulated after the induction of luteinizing hormone or human chorionic gonadotrophin ${ }^{21}$.

To determine which cell types in the mammary gland express miR-212 and miR-132, we isolated mammary epithelial organoids and stromal cells separately and checked the expression of their primary transcript (Supplementary Fig. 1b) and mature forms (Fig. 6a). The purity of isolated fractions was confirmed by quantitative RT-PCR results for vimentin and E-cadherin, which are cell markers for fibroblasts and luminal epithelium, respectively (Fig. 6b). We also analyzed the expression of both microRNAs in the cleared fat pad, from which the epithelium was surgically removed at the pre-pubertal stage and analyzed 5 weeks after the operation. Our results show that cleared fat pad samples contained threefold more fibroblasts and about threefold higher expression of both miR-212 and miR-132 than did 10-week-old mammary gland samples (Fig. 6a). Moreover, the expression of both microRNAs was detected in the mammary stromal cell fractions but not in the mammary epithelial organoids. Therefore, we conclude that miR-212 and miR-132 are expressed exclusively by the stromal cells in the mammary gland.

\section{Structural defects in mutant periductal stroma}

The mammary stroma comprises the fat pad and the periductal stroma. The fat pad contains mainly fibroblasts, preadipocytes, brown adipose tissue and white adipose tissue. The periductal stroma that surrounds the epithelial ducts is mainly composed of fibroblasts and collagen-rich ECM and is essential for the regulation of mammary gland development ${ }^{16,17}$. 
Figure 5 The miR-212/132-/- stroma is not permissive for pubertal outgrowth of the epithelial ducts. (a-h) Whole-mount staining of transplanted whole mammary glands analyzed 5 and 10 weeks after the operation (p.o.). Outlines in $\mathbf{a}, \mathbf{b}$ and $\mathbf{e}-\mathbf{h}$ show locations of the grayscale insets images. Asterisks show the parts of nipple tissues that were also transplanted. Mammary glands from 5-week-old wild-type or heterozygous donor mice had the proper TEBs and ductal outgrowth after being transplanted into homozygous $(\mathbf{a}, \mathbf{c})$ or wild-type $(b, d)$ recipient mice. By contrast, mammary glands of 5-week-old miR-212/132-/- donor mice showed no ductal outgrowth or TEB structures when they were transplanted into miR-212/132--- (e) or wildtype (f) mice. Mammary glands of 3-week-old wildtype mice were also able to initiate ductal outgrowth in $\operatorname{miR}-212 / 132^{-/-}$recipient mice (g), whereas, the glands of 3-week-old mutant mice failed to form proper TEBs in wild-type mice (h). (i) The strategy of the whole mammary gland transplantation. The inguinal (no. 4) glands are shown on both sides of mice in the lower abdomen. Both right and left glands of donor mice were used for transplanting, each into a different recipient mouse (arrows). The location where the transplants were placed is shown in the upper abdomen in a perpendicular orientation to the endogenous inguinal glands. Arrows in $\mathbf{a}, \mathbf{b}$ and $\mathbf{g}$ show TEBs on the distal tips of primary ducts. Scale bars represent $1 \mathrm{~cm}$.

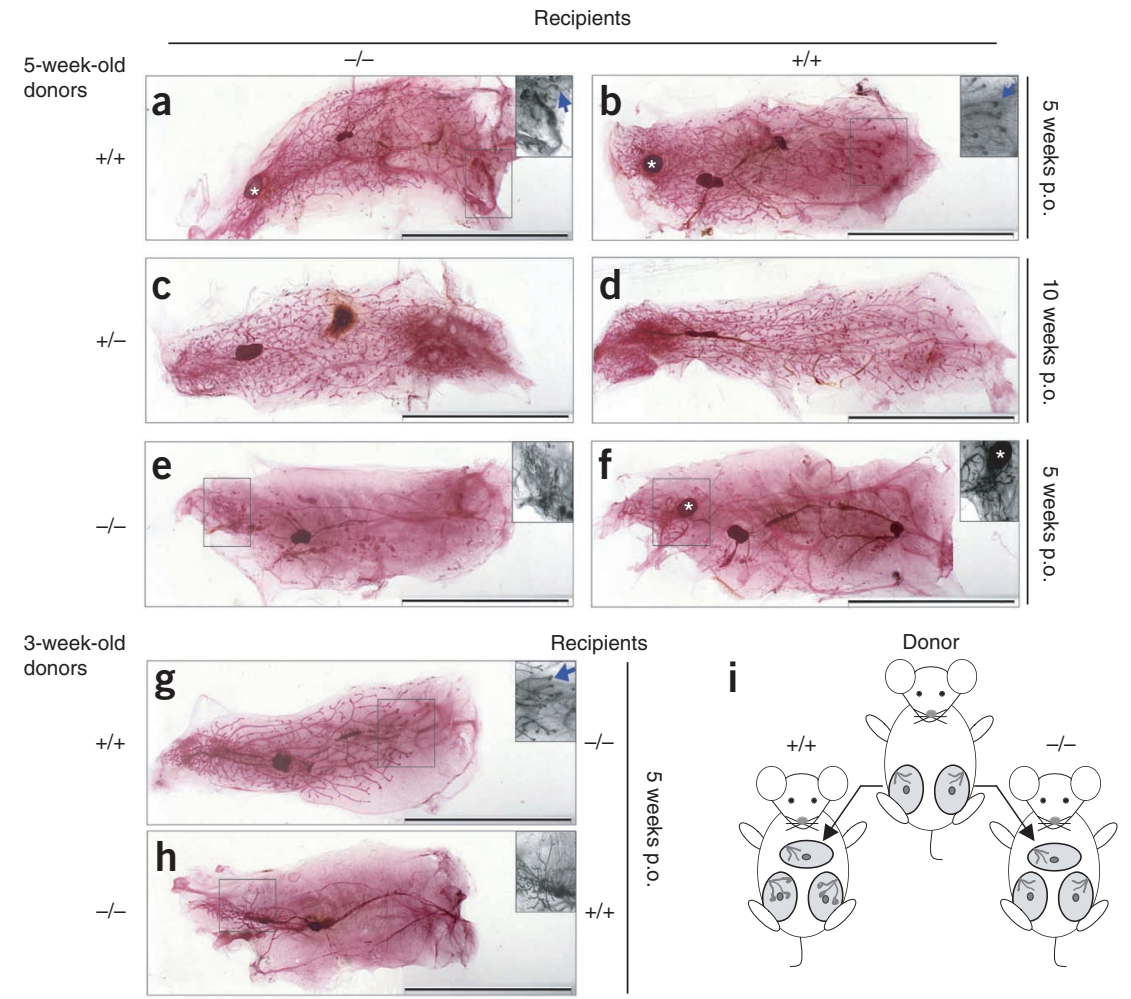

As miR-212 and miR-132 were exclusively expressed in the mammary stroma and a stromal defect caused the impaired ductal outgrowth phenotype in $m i R-212 / 132^{-l-}$ glands, we investigated the integrity of the periductal stroma in mutant glands by Masson's trichrome staining.

The periductal stroma is rich in fibroblasts around the neck region of TEBs and in the distalmost ducts (Supplementary Fig. 6a,b). In these regions, there are low levels of collagen. However, more proximal primary ducts are generally surrounded with collagen-rich periductal stroma but are associated with fewer fibroblasts (Supplementary Fig. 6c). We did not detect such collagen-rich periductal stroma around the proximal or relatively distal ducts of $m i R-212 / 132^{-1-}$ glands, although fibroblasts were still seen close to them (Supplementary Fig. 6d-f). Mutant glands contained blue-staining mucus-like structures in almost every ductal lumen. In relatively distal ducts, it was mostly seen adjacent to the peripheries of the lumen, whereas it covered the lumen entirely in the more proximal ducts (Supplementary Fig. 6d-f).

We also analyzed collagen deposition in transplanted glands composed of wild-type epithelia and $m i R-212 / 132^{-/-}$stroma or vice versa (Supplementary Fig. 7). Transplanted miR-212/132-l- epithelia can form TEBs and undergo ductal outgrowth in the wild-type cleared fat pads (Fig. 4). Mammary ducts formed in such transplants also had similar levels of collagen deposition to the non-transplanted contralateral glands (Supplementary Fig. 7a-c). On the other hand, transplanted wild-type epithelia in mutant cleared fat pads showed impaired ductal outgrowth and also showed the same collagen deposition defect as observed in the non-transplanted contralateral glands (Supplementary Fig. 7d-f). These results indicate that the collagen deposition defect in $m i R-212 / 132^{-/-}$mammary glands is due to a defect not in the mutant epithelium, but in the mutant stroma.

miR-212 and miR-132 negatively regulate MMP-9 expression The collagen in the periductal stroma of mammary glands is secreted mostly by the epithelia under the control of the tumour growth factor- $\beta$
(TGF- $\beta$ ) pathway ${ }^{22}$. Collagen deposition is also regulated within the ECM by several matrix metalloproteinases (MMPs) and tissue inhibitor of metalloproteinases (TIMPs). MMPs that have a collagenase function degrade the collagen in the ECM, whereas TIMPs specifically inhibit the destructive functions of $\mathrm{MMPs}^{23,24}$. In the mammary gland, these MMPs and TIMPs are produced by epithelia, fibroblasts, mast cells and adipocytes.

MMP-9, also known as collagenase type IV-B, is a predicted target of miR-212 and miR-132 (Miranda database). We tested this bioinformatic prediction using a luciferase assay approach. We cloned the 3' UTR of MMP-9 downstream of the firefly luciferase gene and found that the normalized luciferase activity was substantially reduced upon cotransfection with either miR-212 or miR-132 or with both microRNAs together ('MMP-9' in Supplementary Fig. 8). However, this decrease in luciferase activity was abolished when the binding sites of miR-212 and miR-132 on the $3^{\prime}$ UTR of MMP-9 were mutated in the luciferase construct ('MMP-9-mut'. in Supplementary Fig. 8). These results indicate that MMP-9 is a direct target of both miR-212 and miR-132.

Previous in situ hybridization data showed that MMP-9 is expressed at low levels throughout the mammary gland in the epithelia and stroma, but high levels of MMP-9 mRNA were also detected in some cells scattered within the fat $\mathrm{pad}^{25}$. We used immunohistochemistry to analyze MMP-9 expression in both wild-type and $m i R-212 / 132^{-/-}$mammary glands.

In wild-type glands, we detected low levels of MMP-9 throughout the whole fat pad, with slightly higher levels in distal regions than in proximal regions. Around the TEBs, there were several fibroblasts in which MMP-9 was highly expressed (arrows in Fig. 7a). We found low, diffuse staining in the periductal stroma of distal ducts; however, proximal ducts had almost no MMP-9 in their periductal stroma (Fig. 7b,c). Some scattered cells in the fat pad had high MMP-9 levels (blue arrows in Fig. 7c), consistent with the results of the in situ hybridization experiments ${ }^{25}$. By contrast, MMP-9 was much more 
Figure 6 In the mammary gland, miR-212 and miR-132 are exclusively expressed in the stromal cells, but not in the epithelia. (a) Quantitative RT-PCR analyses were performed to detect the expression of miR-212 and miR-132 in 10-week-old whole mammary glands, cleared fat pads and stromal and epithelial organoid fractions from digested glands. Cleared fat pad samples, which are epithelium-free, have a three-fold higher expression of miR-212 and miR-132 than whole glands. Isolated stromal fractions from mammary glands have the highest expression of both miR-212 and miR-132, whereas isolated epithelial organoids express neither miR-212 nor miR-132. All expression levels were calculated as fold-changes compared to their levels in intact mammary glands from 10-week-old mice. (b) Quantitative RT-PCR analyses were performed for E-cadherin and vimentin to determine the presence of epithelial and fibroblast cells, respectively, in the same sample set as in a. The absence of E-cadherin expression in cleared fat pad and isolated stromal fraction samples proved that these samples were epithelium-free. The absence of vimentin expression in isolated epithelial organoids also showed that there was no fibroblast contamination in their preparation. Data are expressed as mean \pm s.d.; $n=3$ for each sample set.

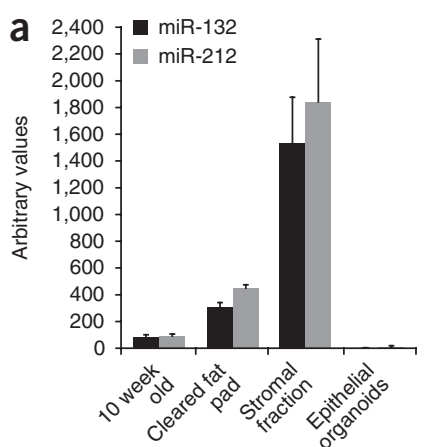

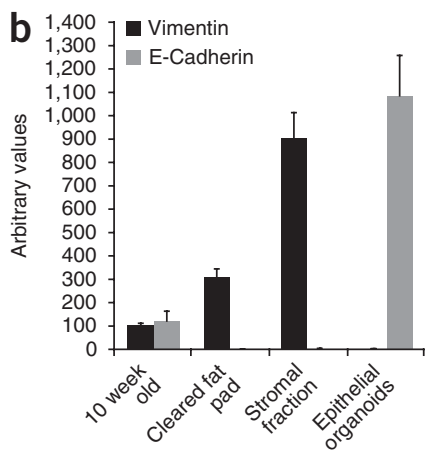

highly expressed throughout the $m i R-212 / 132^{-/-}$glands. All epithelial ducts contained high levels of diffuse MMP-9 staining in their periductal stroma (Fig. 7d-f). In addition, we found fibroblasts that expressed MMP-9 in the periductal stroma of mutant ducts (black arrows in Fig. 7f), similar to the fibroblasts observed around the neck region of TEBs in wild-type glands (Fig. 7a). Also within the fatty stroma, there were many scattered cells, presumably fibroblasts, that contained high concentrations of MMP-9 (blue arrows in Fig. 7f). These results indicate that in the absence of negative regulation by miR-212 and miR-132, the expression of MMP-9 in stromal cells is markedly increased. The accumulation of MMP-9 in the periductal stroma might explain the collagen deposition defect in $m i R-212 / 132^{-/-}$glands.

\section{Hyperactivation of TGF- $\beta$ pathway in $m i R-212 / 132^{-/-}$glands} TGF- $\beta$ regulates ductal outgrowth and side branching during mammary gland development. ${ }^{23,24}$ Overexpression of TGF- $\beta$ in pubertal mammary glands causes impairment of ductal outgrowth and results in simplified arborization patterns of ducts ${ }^{23,26}$. All three isoforms of TGF- $\beta$ are expressed in mammary epithelia with different spatiotemporal distributions ${ }^{23,26}$. The TGF- $\beta$ pathway suppresses the proliferation of epithelial cells and regulates the synthesis of ECM proteins by these cells.

All three isoforms of TGF- $\beta$ bind to the same TGF- $\beta$ receptors and activate downstream pathways through the phosphorylation of Smad 2 and Smad3 (ref. 27). However, TGF- $\beta$ is secreted in a latent form as covalently associated with the latency-associated polypeptide (LAP) ${ }^{23,27}$. Within the ECM, latent TGF- $\beta$-binding proteins (LTBPs) also bind to the LAP-TGF- $\beta$ complexes to keep TGF- $\beta$ in a latent state ${ }^{27}$. TGF- $\beta$ accumulates in the periductal stroma within the mammary glands ${ }^{23}$. It is believed that the collagenrich ECM is important for maintaining a reservoir of latent TGF- $\beta$ around the epithelial ducts and also for keeping TGF- $\beta$ in a latent
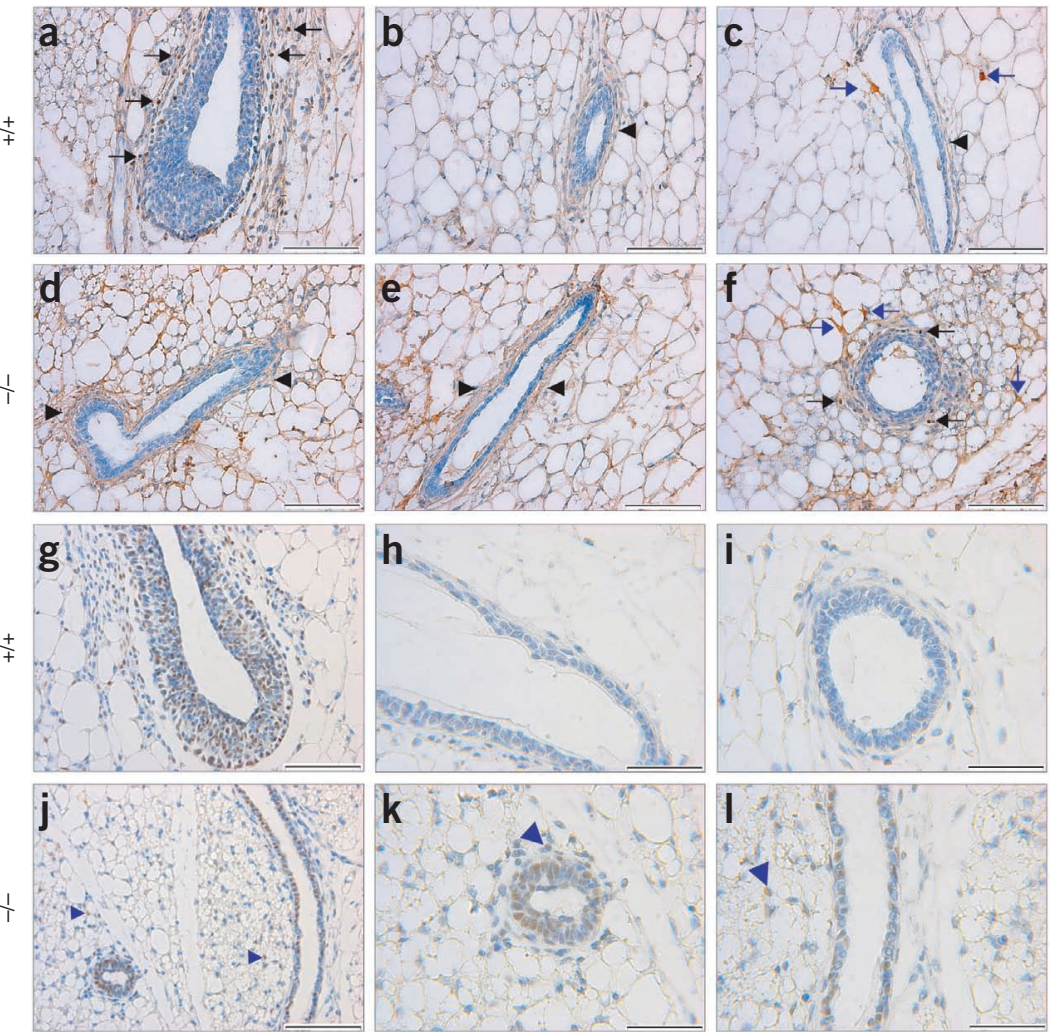

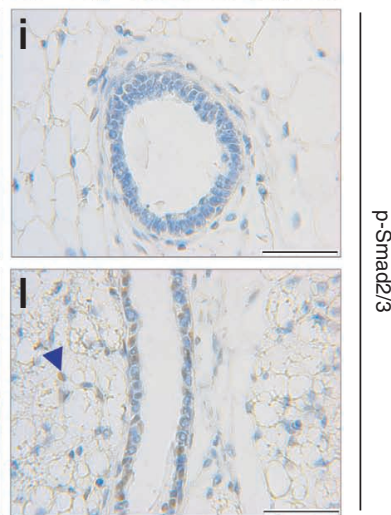

Figure 7 Loss of miR-212 and miR-132 causes high MMP-9 levels and hyperactivity of TGF- $\beta$ pathway in mutant mammary glands. Immunohistochemical staining for MMP-9 (a-f) and phosphorylated Smad2 or 3 ( $p-S m a d 2 / 3$; g-I) on mammary gland sections from 6-weekold wild-type mice (a-c, $\mathbf{g}-\mathbf{i})$ and homozygous mutant littermates (d-f, $\mathbf{j}-\mathbf{l}$ ). In wild-type mammary glands, high MMP-9 was seen only in the periductal stroma of TEBs. Arrows show the MMP-9-expressing fibroblasts in a. The periductal stroma of growth-quiescent ducts in wild-type glands has low MMP-9 staining (arrowheads in $\mathbf{b}$ and $\mathbf{c}$ ). In the fat pad between ducts, there are a few scattered fibroblasts expressing high levels of MMP-9 (blue arrows in c). The periductal stroma in mutant glands shows high MMP-9 (arrowheads in $\mathbf{d}$ and $\mathbf{e}$ ), and both periductal stroma and fat pad contain many MMP-9-expressing fibroblasts (black and blue arrows, respectively, in f). In wildtype mammary glands, $\mathrm{p}$-Smad2/3 is present in the epithelial cells of TEBs and fibroblasts around TEBs (g). However, $\mathrm{p}-\mathrm{Smad} 2 / 3$ is not detectable in the epithelia or stroma of growthquiescent ducts of wild-type glands $(\mathbf{h}, \mathbf{i})$. In mutant glands, most epithelial cells of all ducts are stained for $p-S m a d 2 / 3(j-I)$. There were also several p-Smad2/3-positive fibroblasts in both periductal stroma and fat pads of mutant glands (blue arrowheads in $\mathbf{j}-\mathbf{I}$ ). Scale bars represent $50 \mu \mathrm{m}$ for $\mathbf{h}, \mathbf{i}, \mathbf{k}, \mathbf{l}$ and $100 \mu \mathrm{m}$ for other panels. 
form by making it inaccessible to the proteins or environmental conditions that are necessary for its activation ${ }^{23,24}$.

MMP-9 can activate latent TGF- $\beta$ by dissociating the latency complex that contains TGF- $\beta$, LAP and LTBPs ${ }^{28,29}$. Therefore, high levels of MMP-9 and a marked reduction in collagen in the periductal stroma might result in the hyperactivation of latent TGF- $\beta$ around the miR-212/132 ${ }^{-/}$mammary ducts. To test this hypothesis, we performed immunohistochemical analyses with an antibody that recognizes only the phosphorylated forms of both Smad2 and Smad3. The presence of phosphorylated Smad2 or Smad3 in a cell is a strong indicator for the activation of the TGF- $\beta$ pathway. In wild-type mammary glands, the majority of epithelia and fibroblasts in the proliferative zones, such as TEBs (Fig. 7g) and ductal side-buds (data not shown), were positive for phosphoSmad2 or phospho-Smad3. By contrast, in the growth-quiescent ductal regions of wild-type glands, almost no epithelia or fibroblasts showed detectable staining for phospho-Smad2 or phospho-Smad3 (Fig. 7h,i). However, in $m i R-212 / 132^{-l-}$ glands, most of the epithelia in all ductal regions contained high levels of phospho-Smad 2 or phospho-Smad3, indicating that the TGF- $\beta$ pathway was highly active (Fig. 7j-1). We also found several fibroblasts that contained phospho-Smad2 or phospho-Smad3 in the mutant stroma around the ducts (blue arrowheads in Fig. $7 \mathbf{j}-\mathbf{1}$ ).

The main molecular pathway that is known to induce epithelial proliferation in pubertal mammary glands involves the transcriptional activation of the amphiregulin (Areg) gene by the estrogen receptor $\mathrm{ER} \alpha$ in a manner that depends on the transcriptional cofactor Cited1 (ref. 11). TGF- $\beta$ negatively regulates this pathway to suppress epithelial proliferation ${ }^{11}$. Therefore, we checked the levels of Cited 1 and Areg mRNA in pubertal mammary glands and found that they were both significantly $(P<0.005)$ downregulated in $m i R-212 / 132^{-/-}$ glands (Supplementary Fig. 9). This provides additional evidence that the TGF- $\beta$ pathway is hyperactivated in $m i R-212 / 132^{-/-}$mammary glands.

To find out whether there is any deficiency of ER $\alpha$ expression in $m i R-212 / 132^{-1-}$ mammary glands, which might also cause the downregulation of Areg expression, we checked its expression pattern by imunohistochemistry (Supplementary Fig. 10). The expression of ER $\alpha$ in both epithelia and stroma of $m i R-212 / 132^{-/-}$mammary glands was similar to that seen in wild-type glands. These results indicate that the downregulation of Areg mRNA in miR-212/132-/glands is not due to a defect in ER $\alpha$ expression, but might be due to the hyperactivation of the TGF- $\beta$ pathway.

Together, our findings indicate that in the absence of miR-212 and miR-132, MMP-9 levels increase in the mutant mammary stroma, which probably causes the defect in collagen deposition and the hyperactivation of the TGF- $\beta$ pathway. High TGF- $\beta$ activity can downregulate Cited 1 mRNA, thereby preventing the transcriptional activation of Areg in mammary epithelia, which in turn leads to impaired ductal outgrowth in $m i R-212 / 132^{-1-}$ mammary glands.

\section{DISCUSSION}

We have shown that the miR-212/132 family is required and indispensable for proper ductal outgrowth during pubertal development of the mammary glands in mice. Transplantation experiments showed that the impaired ductal outgrowth in $m i R-212 / 132^{-/-}$ mammary glands was caused by defects not in the epithelia but in the stroma. Levels of circulating estrogen and growth hormone were normal in $m i R-212 / 132^{-/-}$mice. miR-212 and miR-132 were exclusively expressed by stromal cells in the mammary gland, and they directly downregulated the expression of MMP-9. In the absence of regulation by miR-212 and miR-132, MMP-9 increased and accumulated in the periductal stroma of mutant glands. The observed collagen deposition defects in $m i R-212 / 132^{-/-}$glands were probably due to this increase in MMP-9. The periductal stroma accommodates a large reservoir of latent TGF- $\beta$, which is necessarily kept in an inactive state within the collagen-rich ECM ${ }^{23}$. As MMP-9 can activate latent TGF- $\beta^{28,29}$, the absence of collagen and high MMP-9 levels in mutant periductal stroma may eventually cause hyperactivation of latent TGF- $\beta$. Consistent with this model, we have shown that the TGF- $\beta$ pathway was hyperactivated in $m i R-212 / 132^{-/-}$mammary glands.

The TGF- $\beta$ pathway positively regulates both the production and secretion of MMP-9 in various cell types ${ }^{30-34}$. Such possible upregulation of MMP-9 expression by TGF- $\beta$ might generate a positive feedback loop in miR-212/132-/- mammary glands, as MMP-9 can also activate the latent TGF- $\beta^{28,29}$. Therefore, the essential function of miR-212 and miR-132 might be to suppress this positive feedback loop, which otherwise might be deleterious for collagen-rich ECM formation around the mammary ducts and also might completely suppress ductal outgrowth.

Mice deficient in Cited1, Areg or Egfr (the receptor for amphiregulin) show impaired mammary ductal outgrowth phenotypes similar to that of $m i R-212 / 132^{-/-}$mice ${ }^{35-38}$. The downregulation of the expression of Cited 1 and amphiregulin in $m i R-212 / 132^{-1-}$ glands might explain the similarity in the observed phenotypes. Cited 1 also acts as a cofactor for Smad 4 in the TGF- $\beta$ pathway ${ }^{39}$. Therefore, high TGF- $\beta$ activity in miR-212/132-/- epithelia might selectively regulate the expression of a specific set of genes, whose regulation would not depend on the interaction between Cited 1 and Smad4.

The signaling proteins Wnt5a and Fgfr2b are also downregulated in miR-212/132 $2^{-1}$ mammary glands (our unpublished microarray data). As Fgfr2b is essential for TEB maintenance ${ }^{40,41}$, its downregulation might also explain the absence of TEBs in $m i R-212 / 132^{-1-}$ glands. Wnt5a is the main downstream mediator in the suppression of ductal outgrowth by TGF- $\beta^{42}$. Its downregulation in $m i R-212 / 132^{-/-}$glands might be explained by the possibility that its transcriptional control depends on the interaction between Smad4 and Cited1. However, in the absence of Wnt5a upregulation, ductal outgrowth is still impaired in $m i R-212 / 132^{-/-}$glands, suggesting that other mediator(s) of the TGF-B pathway also regulate ductal outgrowth.

Previous studies showed the functional importance of other microRNAs in mammary epithelial cell lines ${ }^{43-45}$. However, our results provide the first in vivo example of microRNA function being required for mammary gland development. Moreover, we have shown that microRNAs not only regulate autonomous cell functions, but also are central to communication between stromal and epithelial cells.

URLs. Miranda database, http://www.microrna.org/.

\section{METHODS}

Methods and any associated references are available in the online version of the paper at http://www.nature.com/naturegenetics/.

Accession codes. The full-length sequence of primary transcript encoding the transcript variant 2 of $m i R-212 / 132$ gene has been deposited in the GenBank database with the following accession number: HM627212.

Note: Supplementary information is available on the Nature Genetics website.

\section{ACKNOWLEDGMENTS}

We thank S. Geisendorf for technical assistance, S. Hille for DNA sequencing, S. Mahsur and U. Franke for technical help in generation of knockout mouse 
line, A. Kurth, A. Driehorst, K. Kiel and U. Teichman for animal care work, M. Smalley for discussion about mammary epithelial transplantation method, N. Ba Tiep for technical help with collecting blood samples, A. Yalcin for creative contribution to the initial phase of the work, and P. Gruss and G. Eichele for encouragement and support. Funded by the Max Planck Society. Additional funding was provided by the Deutsche Forschungsgemeinschaft (DFG, GR 536/9-2 and GR 536/11-1 to B.G. and TH 903/7-2 to T.T.).

\section{AUTHOR CONTRIBUTIONS}

A.U. and K.C. developed the concept of this study. A.U. performed all experiments except the mammary epithelial transplantation analyses, which were performed by V.V. and P.A.B.K. in the laboratory of B.G., serum hormone level analyses, which were done by H.J., and luciferase assays, which were done by J.F. in the laboratory of T.T. The manuscript was written by A.U. and K.C. Important suggestions were made by B.G., V.V. and T.T. which improved the quality of the manuscript. All authors contributed to the discussion of the data and commented on the final version of the manuscript.

\section{COMPETING FINANCIAL INTERESTS}

The authors declare no competing financial interests.

Published online at http://www.nature.com/naturegenetics/.

Reprints and permissions information is available online at http://npg.nature.com/ reprintsandpermissions/.

1. Valencia-Sanchez, M.A., Liu, J., Hannon, G.J. \& Parker, R. Control of translation and mRNA degradation by miRNAs and siRNAs. Genes Dev. 20, 515-524 (2006).

2. Bartel, D.P. MicroRNAs: Target recognition and regulatory functions. Cell 136 , 215-233 (2009).

3. Zhao, Y. \& Srivastava, D. A developmental view of microRNA function. Trends Biochem. Sci. 32, 189-197 (2007).

4. Smibert, P. \& Lai, E.C. Lessons from microRNA mutants in worms, flies and mice. Cell Cycle 7, 2500-2508 (2008).

5. Vo, N. et al. A cAMP-response element binding protein-induced microRNA regulates neuronal morphogenesis. Proc. Natl. Acad. Sci. USA 102, 16426-16431 (2005).

6. Wayman, G.A. et al. An activity-regulated microRNA controls dendritic plasticity by down-regulating p250GAP. Proc. Natl. Acad. Sci. USA 105, 9093-9098 (2008).

7. Impey, S. et al. An activity-induced microRNA controls dendritic spine formation by regulating Rac1-PAK signaling. Mol. Cell. Neurosci. 43, 146-156 (2010).

8. Kawashima, H. et al. Glucocorticoid attenuates brain-derived neurotrophic factordependent upregulation of glutamate receptors via the suppression of microRNA132 expression. Neuroscience 165,1301-1311 (2010).

9. Cheng, H.Y. et al. MicroRNA modulation of circadian-clock period and entrainment. Neuron 54, 813-829 (2007).

10. Shaked, I. et al. MicroRNA-132 potentiates cholinergic anti-inflammatory signaling by targeting acetylcholinesterase. Immunity 31, 965-973 (2009).

11. Howlin, J., McBryan, J. \& Martin, F. Pubertal mammary gland development: Insights from mouse models. J. Mammary Gland Biol. Neoplasia 11, 283-297 (2006).

12. Richert, M.M. et al. An atlas of mouse mammary gland development. J. Mammary Gland Biol. Neoplasia 5, 227-241 (2000).

13. Hennighausen, L. \& Robinson, G.W. Signaling pathways in mammary gland development. Dev. Cell 1, 467-475 (2001).

14. Watson, C.J. \& Khaled, W.T. Mammary development in the embryo and adult: a journey of morphogenesis and commitment. Development 135, 995-1003 (2008).

15. Hennighausen, L. \& Robinson, G.W. Information networks in the mammary gland. Nat. Rev. Mol. Cell Biol. 6, 715-725 (2005).

16. Parmar, H. \& Cunha, G.R. Epithelial-stromal interactions in the mouse and human mammary gland in vivo. Endocr. Relat. Cancer 11, 437-458 (2004).

17. Neville, M.C., Medina, D., Monks, J. \& Hovey, R.C. The mammary fat pad. J. Mammary Gland Biol. Neoplasia 3, 109-116 (1998).

18. Wiseman, B.S. \& Werb, Z. Stromal effects on mammary gland development and breast cancer. Science 296, 1046-1049 (2002).

19. Remenyi, J. et al. Regulation of the miR-212/132 locus by MSK1 and CREB in response to neurotrophins. Biochem. J. 428, 281-291 (2010).

20. Mailleux, A.A., Overholtzer, M. \& Brugge, J.S. Lumen formation during mammary epithelial morphogenesis: insights from in vitro and in vivo models. Cell Cycle 7 , 57-62 (2008).
21. Fiedler, S.D., Carletti, M.Z., Hong, X. \& Christenson, L.K. Hormonal regulation of microRNA expression in periovulatory mouse mural granulosa cells. Biol. Reprod. 79, 1030-1037 (2008).

22. Silberstein, G.B., Strickland, P., Coleman, S. \& Daniel, C.W. Epithelium-dependent extracellular matrix synthesis in transforming growth factor-beta 1-growth-inhibited mouse mammary gland. J. Cell Biol. 110, 2209-2219 (1990).

23. Daniel, C.W., Robinson, S. \& Silberstein, G.B. The role of TGF-beta in patterning and growth of the mammary ductal tree. J. Mammary Gland Biol. Neoplasia 1 331-341 (1996).

24. Silberstein, G.B. Postnatal mammary gland morphogenesis. Microsc. Res. Tech. $\mathbf{5 2}$, 155-162 (2001).

25. Wiseman, B.S. et al. Site-specific inductive and inhibitory activities of MMP-2 and MMP-3 orchestrate mammary gland branching morphogenesis. J. Cell Biol. 162, 1123-1133 (2003).

26. Robinson, S.D. et al. Regulated expression and growth inhibitory effects of transforming growth factor-beta isoforms in mouse mammary gland development. Development 113, 867-878 (1991).

27. Kang, J.S., Liu, C. \& Derynck, R. New regulatory mechanisms of TGF-beta receptor function. Trends Cell Biol. 19, 385-394 (2009).

28. Yu, Q. \& Stamenkovic, I. Cell surface-localized matrix metalloproteinase-9 proteolytically activates TGF-beta and promotes tumor invasion and angiogenesis. Genes Dev. 14, 163-176 (2000).

29. Wilson, T.J., Nannuru, K.C. \& Singh, R.K. Cathepsin G-mediated activation of pro-matrix metalloproteinase 9 at the tumor-bone interface promotes transforming growth factor-beta signaling and bone destruction. Mol. Cancer Res. 7, 1224-1233 (2009).

30. Okamoto, $T$. et al. Transforming growth factor-beta 1 induces matrix metalloproteinase-9 expression in human meningeal cells via ERK and Smad pathways. Biochem. Biophys. Res. Commun. 383, 475-479 (2009).

31. Sinpitaksakul, S.N., Pimkhaokham, A., Sanchavanakit, N. \& Pavasant, P. TGF-beta 1 induced MMP-9 expression in HNSCC cell lines via Smad/MLCK pathway. Biochem. Biophys. Res. Commun. 371, 713-718 (2008).

32. Seomun, Y., Kim, J.T. \& Joo, C.K. MMP-14 mediated MMP-9 expression is involved in TGF-beta 1-induced keratinocyte migration. J. Cell. Biochem. 104, 934-941 (2008).

33. Chou, Y.T., Wang, H., Chen, Y., Danielpour, D. \& Yang, Y.C. Cited2 modulates TGFbeta-mediated upregulation of MMP9. Oncogene 25, 5547-5560 (2006).

34. Konrad, L., Scheiber, J.A., Schwarz, L., Schrader, A.J. \& Hofmann, R. TGF-beta1 and TGF-beta2 strongly enhance the secretion of plasminogen activator inhibitor-1 and matrix metalloproteinase-9 of the human prostate cancer cell line PC-3. Regul. Pept. 155, 28-32 (2009).

35. Howlin, J. et al. CITED1 homozygous null mice display aberrant pubertal mammary ductal morphogenesis. Oncogene 25, 1532-1542 (2006).

36. Luetteke, N.C. et al. Targeted inactivation of the EGF and amphiregulin genes reveals distinct roles for EGF receptor ligands in mouse mammary gland development. Development 126, 2739-2750 (1999).

37. Ciarloni, L., Mallepell, S. \& Brisken, C. Amphiregulin is an essential mediator of estrogen receptor alpha function in mammary gland development. Proc. Natl. Acad. Sci. USA 104, 5455-5460 (2007).

38. Wiesen, J.F., Young, P., Werb, Z. \& Cunha, G.R. Signaling through the stromal epidermal growth factor receptor is necessary for mammary ductal development. Development 126, 335-344 (1999).

39. Shioda, T. et al. Transcriptional activating activity of Smad4: roles of SMAD heterooligomerization and enhancement by an associating transactivator. Proc. Natl. Acad. Sci. USA 95, 9785-9790 (1998).

40. Parsa, S. et al. Terminal end bud maintenance in mammary gland is dependent upon FGFR2b signaling. Dev. Biol. 317, 121-131 (2008).

41. Lu, P., Ewald, A.J., Martin, G.R. \& Werb, Z. Genetic mosaic analysis reveals FGF receptor 2 function in terminal end buds during mammary gland branching morphogenesis. Dev. Biol. 321, 77-87 (2008).

42. Roarty, K. \& Serra, R. Wnt5a is required for proper mammary gland development and TGF- $\beta$-mediated inhibition of ductal outgrowth. Development 134, 3929-3939 (2007).

43. Ibarra, I., Erlich, Y., Muthuswamy, S.K., Sachidanandam, R. \& Hannon, G.J. A role for microRNAs in maintenance of mouse mammary epithelial progenitor cells. Genes Dev. 21, 3238-3243 (2007).

44. Kong, W. et al. MicroRNA-155 is regulated by the transforming growth factor beta/ Smad pathway and contributes to epithelial cell plasticity by targeting RhoA. Mol. Cell. Biol. 28, 6773-6784 (2008).

45. Tanaka, T., Haneda, S., Imakawa, K., Sakai, S. \& Nagaoka, K. A microRNA, miR$101 a$, controls mammary gland development by regulating cyclooxygenase-2 expression. Differentiation 77, 181-187 (2009). 


\section{ONLINE METHODS}

Generation of miR-212/132 ${ }^{-/-}$mice. We generated $m i R-212 / 132^{-/-}$mice by homologous recombination targeting the genomic region that encodes both pre-miR-212 and pre-miR-132. Details of the targeting vector and the strategy for obtaining the miR-212/132-I- mouse lines are described in the Supplementary Note. All phenotypical results were obtained from mice of $129 / \mathrm{Sv}$ background. Similar results were also obtained from $m i R-212 / 132^{-/-}$ mice of C57BL6/N background for the whole-mount mammary gland analyses in all pubertal, pregnancy and lactation stages.

RT-PCR analysis. Total RNA samples from dissected tissues or mammary cell fractions were isolated with TRI reagent (Sigma). To detect mature microRNA levels, we carried out reverse transcription (RT) and real-time PCR analyses using TaqMan MicroRNA Reverse Transcription kit (Applied Biosystems) and TaqMan Universal PCR master mix (Applied Biosystems) according to the manufacturer's instructions. Primers for RT and Q-PCR were obtained from TaqMan MicroRNA assay kits for hsa-miR-132, mmu-miR-212, sno-142 and sno-202 (Applied Biosystems). Fold changes are calculated from the determined $\mathrm{Ct}$ values and the expression levels of miR-132 and miR-212 were determined by normalizing these values with the sum of fold change values for sno-142 and sno-212.

For the detection of CITED1 and amphiregulin mRNA levels, cDNAs were synthesized with the ThermoScript RT-PCR System (Invitrogen) using random hexamers according to the manufacturer's instructions. Real-time PCR analyses were performed using iQ SYBR green supermix (Biorad) and QuantiTect Primer assays (Qiagen) for Cited1, Areg, Hprt1 and Actb. Fold changes are calculated from the determined Ct values and the expression levels of Cited 1 and Areg were determined by normalizing these values to the fold-change values for Hprt1 and Actb.

For the detection of specific isoforms of the miR-212/132 gene cDNAs were synthesized as above. PCR primers were designed from exon junctions to prevent any amplification from contaminating genomic DNA within the RNA samples (Supplementary Table 1). RT (-) controls were also included in the experiments.

Whole-mount analysis of mammary glands. Whole-mount carmine-alum staining of mammary glands was performed as described ${ }^{46}$. Briefly, inguinal (no. 4) mammary glands were dissected from mice, spread onto glass slides, fixed in a 6:3:1 mixture of ethanol:chloroform:glacial acetic acid, hydrated, stained overnight in $0.2 \%$ carmine (Sigma) and $0.5 \% \mathrm{AlK}\left(\mathrm{SO}_{4}\right)_{2}$, dehydrated in graded solutions of ethanol, cleared in Histoclear and mounted.

Animal studies. All animal studies were performed in accordance with the relevant guidelines and regulations and with the approval of the responsible local and national authorities. For the pup growth curve analysis, female littermates were mated and the number of pups per litter was reduced to five after parturition. Litters containing fewer than five pups were not used. The pup weights were recorded during the indicated postnatal days of life. For hormone analysis in blood serum, mice were anaesthetized and the blood samples were collected by retro-orbital puncture.

Mammary epithelial transplantation. Reciprocal mammary epithelial transplantations were done with 3-week-old female littermates (Fig. 4a). Fat pad clearing and transplantations of epithelial ducts were done as described ${ }^{47}$. Briefly, mice were anaesthetized and inguinal mammary glands were exposed through small incisions in the skin of the lower abdomen and along the right hind leg. For fat pad clearing, the connection between glands no. 4 and 5 was disrupted by cauterization and the endogenous epithelia were removed by removing the region between the nipple and the line above the lymph node. Small pieces of the removed glands $\left(\sim 2 \mathrm{~mm}^{3}\right.$ and close to the nipple region) were cut and transplanted into the cleared fat pads of recipient mice. The left inguinal (no. 4) mammary glands were used as controls. For analyses of ductal outgrowth, transplanted mice were killed 3, 12 or 25 weeks after the operation. For the determination of alveolar differentiation, the transplanted mice were mated 2 months after the operation and killed within the first $6 \mathrm{~h}$ post-partum. From these mice, both transplanted and contralateral endogenous control glands were dissected and either analyzed by whole-mount carmine-alum staining or embedded into paraffin for Masson's trichrome staining. In total, 22 sets of reciprocal transplantations were done and reproducible results were obtained from all of them.

Whole mammary gland transplantation. These transplantations were done as described ${ }^{48}$. Briefly, both right and left inguinal (no. 4) mammary glands were dissected from killed donor mice and kept for a short time in DMEM until transplantation. Meanwhile, recipient mice were anaesthetized and small incisions were made in the skin of the upper abdomen. The transplants were placed on the peritoneal wall just under the sternum as shown in Figure 5i. Transplanted and endogenous inguinal glands were dissected 5 or 10 weeks after the operation and analyzed by whole mount carmine-alum staining. At least three sets of transplantation were done for each experimental condition and reproducible results were obtained from all of them.

Histological staining. Dissected mammary glands were fixed in $4 \%$ paraformaldehyde and then either frozen in cryomatrix or embedded in paraffin. Cryosections $(25 \mu \mathrm{m})$ were used for neutral-red staining. Paraffin sections $(7 \mu \mathrm{m})$ were used for Masson's trichrome staining, hematoxylin-eosin staining or immunohistochemistry. Trichrome staining was performed using the Masson's trichrome staining kit (Diagnostic Biosystems) according to the manufacturer's instructions. For immunohistochemical detection of proteins, tissue sections were treated with $3 \% \mathrm{H}_{2} \mathrm{O}_{2}$ in methanol for $10 \mathrm{~min}$, followed by boiling in $0.1 \mathrm{mM}$ citrate buffer ( $\mathrm{pH}$ 6.0) for $15 \mathrm{~min}$ for antigen retrieval. The sections were blocked in goat serum and then primary antibodies for E-cadherin (Santa Cruz Biotechnology, sc-7870, 1:50 dilution), cytokeratin-14 (Abcam, ab53115, 1:100 dilution), MMP-9 (Abcam, ab38898 1:500 dilution), phospho-Smad2 (Cell Signaling Technology, \#3101, 1:200 dilution) or ER $\alpha$ (Abcam, ab37438, 1:50 dilution) were applied. For detection of primary antibodies, the ABC staining system (Santa Cruz Biotechnology, sc-2018) was used according to the manufacturer's instructions.

Separation of mammary stromal fractions and epithelial organoids. Twenty wild-type female mice (8-12-weeks-old) were used for the isolation of epithelial organoids and mammary stromal fractions. Inguinal glands (no. 4) were dissected by excluding the lymph nodes. Afterwards, they were minced into small pieces $\left(\sim 1 \mathrm{~mm}^{3}\right)$ and lysed in $10 \mathrm{ml}$ of digestion cocktail $(1.5 \mathrm{mg} / \mathrm{ml}$ trypsin, $3 \mathrm{mg} / \mathrm{ml}$ collagenase A, 10\% FCS in Ham's F12 medium) for $2 \mathrm{~h}$ at $37^{\circ} \mathrm{C}$ with slow stirring. DNAse (final concentration of $0.1 \mathrm{mg} / \mathrm{ml}$ ) was added and the samples incubated for $10 \mathrm{~min}$ at room temperature. Lysates were left to settle and the floating fatty layer was discarded. Epithelial organoids accumulated in the bottom of the tube. The supernatant was carefully collected into a new tube as the stromal fraction and centrifuged at $500 \mathrm{~g}$, and the pellet was frozen for RNA isolation. The remaining portion of the lysate, containing epithelial organoids, was washed five times with Ham's F12 medium by centrifugation at $10 \mathrm{~g}$ with supernatants discarded to remove remaining fibroblasts from epithelial organoids. Purified epithelial organoids were collected and frozen for RNA isolation.

Serum analyses. Estradiol in the blood serum was measured using the UltraSensitive Estradiol kit (DSL) according to the manufacturer's guidelines. Growth hormone in the blood serum was measured by using the specific radioimmunoassay (RIA) and the standard protocol supplied by the National Hormone and Pituitary Program of the NIDDK. Briefly, $50 \mu 1$ serum was added to $200 \mu \mathrm{l}$ rabbit anti-GH antibody $(1: 5,000)$ and incubated overnight at $4{ }^{\circ} \mathrm{C}$. Subsequently, $100 \mu{ }^{125} \mathrm{I}$-labeled tracer (20,000 c.p.m.) was added and samples were incubated for $24 \mathrm{~h}$ at $4{ }^{\circ} \mathrm{C}$. After the addition of the secondary antibody (sheep anti-rabbit, 1:30), samples were incubated for $48 \mathrm{~h}$ at $4{ }^{\circ} \mathrm{C}$ and finally centrifuged for $1 \mathrm{~h}$ at 2,400g. Radioactivity in the pellets was counted with a Wallac-LKB 1270 counter and analyzed with the RIA-CALC program.

Luciferase assay. A 398-bp fragment from the 3' UTR of MMP-9, containing predicted binding sites for miR-212 and miR-132, was amplified by PCR and cloned as the 'MMP-9' construct into the 3 ' UTR of the luc gene in the pMIR-Report plasmid (Ambion). For the 'MMP-9 mut' construct, mutations were generated 
in the target site of the seed regions of miR-212 and miR-132 by site-directed mutagenesis using the same 398-bp fragment and subsequently cloned into the same region in the pMIR-Report plasmid. The correct DNA sequences were confirmed by sequencing. The cloned constructs were cotransfected with miRNAs of interest (Ambion) and $\beta$-galactosidase control plasmid (Ambion) into HEK293 reporter cells seeded in 48-well plates using Lipofectamine2000 (Invitrogen). In each case, $0.2 \mu \mathrm{g}$ plasmid DNA, $100 \mathrm{nM}$ miRNA and $0.5 \mu \mathrm{l}$ Lipofectamine2000 were applied. Cells were incubated for $24 \mathrm{~h}$ before luciferase and $\beta$-galactosidase activity were measured using the Luciferase Assay System (Promega) and Beta-Galactosidase Assay system (Promega) kits on a multi-plate reader (Biotek, Synergy HT) according to the manufacturers' instructions.
Statistics. Data are expressed as mean \pm s.d. or as median \pm s.e.m. Statistical comparisons were tested by Student's $t$-test using Graphpad Prism software. $P<0.05$ was considered statistically significant.

46. Rasmussen, S.B., Young, L.J.T. \& Smith, G.H. in Methods in Mammary Gland Biology and Breast Cancer Research. (eds. Ip, M.M. and Asch, B.B.) 75-86 (Kluwer Academic/Plenum, New York, 2000).

47. Young, L.J.T. in Methods in Mammary Gland Biology and Breast Cancer Research (eds. Ip, M.M. and Asch, B.B.) 67-74 (Kluwer Academic/Plenum, New York, 2000).

48. Sangai, T. et al. Hormonal stimulation increases the recruitment of bone marrowderived myoepithelial cells and periductal fibroblasts into the mammary gland. Biochem. Biophys. Res. Commun. 346, 1173-1180 (2006). 